\title{
Processing lexical ambiguity and visual word recognition in a deep orthography
}

\author{
SHLOMO BENTIN \\ Hadassah Hospital, Jerusalem, Israel, and Hebrew University, Jerusalem, Israel
}

and

RAM FROST

Hebrew University, Jerusalem, Israel

\begin{abstract}
We investigated the effect of semantic and phonemic ambiguity on lexical decision and naming performance in the deep Hebrew orthography. Experiment 1 revealed that lexical decisions for ambiguous consonant strings are faster than those for any of the high- or low-frequency voweled alternative meanings of the same strings. These results suggested that lexical decisions for phonemically and semantically ambiguous Hebrew consonant strings are based on the ambiguous orthographic information. However, a significant frequency effect for both ambiguous and unambiguous words suggested that if vowels are present, subjects do not ignore them completely while making lexical decisions. Experiment 2 revealed that naming low-frequency voweled alternatives of ambiguous strings took significantly longer than naming the high-frequency alternatives or the unvoweled strings without a significant difference between the latter two string types. Voweled and unvoweled unambiguous strings, however, were named equally fast. We propose that semantic and phonological disambiguation of unvoweled words in Hebrew is achieved in parallel to the lexical decision, but is not required by it. Naming Hebrew words usually requires a readout of phonemic information from the lexicon.
\end{abstract}

This study examined the influence of phonemic and semantic ambiguity on lexical decision and naming performance in Hebrew. Most lexical ambiguity research has concentrated primarily on the way homographs (polysemous words) are disambiguated within a semantic context (for a recent review, see Simpson, 1984). Little has been said about lexical access of isolated ambiguous words or about ambiguity effects on the process of word recognition.

Examination of lexical ambiguity in relation to lexical access and models of word recognition is of special interest in Hebrew orthography because of its special way of conveying phonemic information. In Hebrew, the vowels are signified by small diacritical dots and dashes, and letters carry mostly consonantal information. In most printed texts (e.g., books, magazines, newspapers), the diacritical vowel marks are omitted. (For a detailed description of Hebrew orthography, see Navon \& Shimron, 1984.) Because several words may share an identical consonantal

We thank Morris Moscovitch, Leonard Katz, and James Chumbley for useful comments on earlier drafts of this paper, and Tammi Rossman for her skillful help. This work was supported in part by National Institute of Child Health and Human Development Grant HD-01994 to Haskins Laboratories.

The first author is affiliated with the Aranne Laboratory of Human Psychophysiology, Department of Neurology, Hadassah Hospital, and both authors are affiliated with the Department of Psychology, Hebrew University. Address correspondence to Shlomo Bentin, Aranne Laboratory of Human Psychophysiology, Department of Neurology, Hadassah Hospital, P.O. Box 12000, Jerusalem 91120, Israel. structure, an unequivocal lexical (and phonemic) representation can be attributed to a letter string only through a top-down process, which is usually affected by semantic context. In other words, homographs are the rule rather than the exception in Hebrew, and processing lexical ambiguity is a routine procedure for the reader. The nature of ambiguity in Hebrew consonant strings is different, however, from that in English homographs. Ambiguity in Hebrew print results from missing vowel information. Thus, in contrast to English, which, with a few exceptions (see, e.g., Carpenter \& Daneman, 1981) most homographs are also homophones, Hebrew consonant strings are not only semantically but also phonemically ambiguous. Moreover, the number of different etymological derivations represented by one letter string is usually much higher in Hebrew than in English. Consequently, there is greater uncertainty about which word is represented by a given consonant string in Hebrew than there is about an English homograph.

It is because of this high level of uncertainty that we assumed that lexical decisions for Hebrew homographs might be made without phonological disambiguation. Rather, it is possible that an abstract orthographic representation, common to all alternative meanings of a consonant string, provides, in most cases, the necessary and sufficient information for the lexical decision.

Previous research has already suggested that lexical decisions in Hebrew are based primarily on graphemic/orthographic cues (Bentin, Bargai, \& Katz, 1984; 
Koriat, 1984), but the nature of this process has never been elaborated. One possible assumption is that although lexical access is mediated primarily by orthographic codes, the decision is delayed until one phonological alternative is determined. Thus, a specific word must be deciphered from print before the positive decision can be generated. A second possibility is that a positive decision can be based on the phonologically ambiguous homographic cluster common to all alternatives, and that lexical disambiguation is a subsequent process. We attempted to investigate the validity of each of these assumptions by comparing lexical decision performance on Hebrew consonant strings in the unvoweled ambiguous form and those disambiguated by the vowel marks. ${ }^{1}$

Early studies revealed that, in English, lexical decisions for homographs are faster than for nonhomographs $(H$. Rubenstein, Garfield, \& Millikan, 1970; H. Rubenstein, Lewis, \& M. A. Rubenstein, 1971). One common explanation for this effect of homography is that words with multiple meanings have multiple entries, and therefore the probability of encountering, during lexical search, one of many entries of a word with a large number of meanings is greater than the probability of encountering the single entry of a word with only one meaning. Recently, Jastrzembski (1981) elaborated on this model on the basis of Morton's $(1970,1979)$ logogen theory, assuming that words having multiple meanings are represented by one logogen for each meaning. Jastrzembski assumed that logogens accumulate evidence in a probabilistic fashion. Thus, the more logogens a word has, the more likely one of them will reach threshold. Accordingly, a word with many meanings, and therefore many logogens, will be more likely to have one of its logogens reach threshold sooner than a word with few logogens. Note, however, that all the probabilistic models of lexical access that were proposed to account for the effect of homography were based on responses to homographs that were also homophones. In contrast, it has been reported that, in English, the latency of naming homographs that have different pronunciations for different meanings (e.g., wind) is significantly longer than that of naming homographs with a single pronunciation (e.g., fall) (Kroll \& Schweickert, 1978).

These data suggest that, in English, phonological ambiguity must be resolved before the lexical decision is generated. If the same strategy is employed in Hebrew, lexical decisions about unvoweled, phonologically ambiguous strings should be slower than decisions about any of their explicitly voweled, phonologically disambiguated alternatives. On the other hand, faster responses to the ambiguous homograph than to any of its disambiguated alternatives would suggest that phonological disambiguation is not required for lexical decision. We will claim that such a result supports the possibility that lexical decisions for phonologically ambiguous consonant strings are based primarily on the abstract orthographic representation, which is common to all phonological alternatives. In other words, such a result might suggest that the differ- ent phonological representations of a Hebrew consonant string are related to only one visual logogen.

\section{EXPERIMENT 1}

Experiment 1 compared lexical decision performance for unvoweled ambiguous consonant strings and for their voweled disambiguated alternatives. Each ambiguous consonant string could represent either a high-frequency or a low-frequency word. The same voweled words were presented in two task conditions that were determined by the nature of the nonwords employed. In the optional condition, the nonwords were legal but meaningless permutations of the consonantal letters of real words. In this condition, words and nonwords were distinguishable simply on the basis of the consonantal pattern, so that there was no logical necessity for the subject to attend to the vowels in order to make a lexical decision. In the obligatory condition, special nonwords were used. The consonantal pattern of these nonwords corresponded to real words in Hebrew, but they were presented in an inappropriately voweled manner. Thus, the special nonwords employed in the obligatory condition were in fact words when unvoweled or when voweled correctly; therefore, in this condition, the subject was forced to attend to the vowels, without which discrimination between words and nonwords could not have been made. Recall that the difference between the high- and low-frequency alternatives of each homograph was indicated in print only by the vowel marks. Therefore, the relative sizes of the frequency effect in the optional and in the obligatory condition may reflect the extent to which the information provided by the vowels was processed in each of these conditions by subjects while generating the lexical decision.

In addition to comparing performance for ambiguous strings, Experiment 1 also compared lexical decision performance for voweled and unvoweled unambiguous words. Unambiguous words were consonant strings that represented only one legal phonological derivation. Thus, although the phonology presented in print was not complete if vowel marks were missing, access to these words did not require a choice between different phonological alternatives. Therefore, any difference between voweled and unvoweled unambiguous words should reflect only the effect of missing phonemic information, but no effects of ambiguity. Comparison of lexical decision performance for ambiguous and unambiguous words in the optional and obligatory conditions is particularly important because, regardless of the ambiguity of the words, different nonwords might differently affect performance on low- and high-frequency words (Duchek \& Neely, 1984, cited by Balota \& Chumbley, 1984; James, 1975).

If lexical decisions for unvoweled and for voweled words with ambiguous consonant strings in the optional condition are based primarily on the ambiguous consonantal information, then (1) the reaction times (RTs) for unvoweled ambiguous words should not be longer than RTs 
for any of their voweled high- and low-frequency disambiguated alternatives, and (2) word frequency should have a larger effect in the obligatory than in the optional condition (since subjects in the obligatory condition were forced to process the vowels in order to discriminate between words and nonwords). On the other hand, if phonological disambiguation is mandatory for lexical decisions and if positive responses for Hebrew homographs are based on successful access to an entry uniquely related to one alternate meaning, then (1) RTs for the unvoweled words should be longer than for the voweled words (since vowels simplify the phonological disambiguation of the ambiguous consonant string), and (2) word frequency should have a similar effect on lexical decisions for ambiguous and unambiguous words in the optional and in the obligatory conditions.

\section{Method}

Subjects. Ninety-six undergraduate students either participated as part of the requirements of an introductory psychology course or were paid for their participation. They were all native speakers of Hebrew with normal or corrected-to-normal vision.

Stimuli. The ambiguous words were 16 unvoweled consonant strings. Each string represented a pair of different nouns with different meanings, different pronunciations, and different word frequencies. Lexical decision performance for each of these unvoweled strings was compared with lexical decision performance for its two voweled alternate representations (Figure 1). The 16 pairs of nouns were selected from a pool of 50 similar pairs on the basis of frequency evaluation, as follows. The 100 words were printed with the vowel marks on a single page in random order. Fifty undergraduates were asked to rate each word on a 5-point scale ranging from least frequent (1) to most frequent (5). Estimated frequency for each word was calculated by averaging the rating across all 50 judges. Words rated above 3.5 and below 2.5 were included in the high- and low-frequency groups, respectively. The 16 word pairs selected for this study were those in which one member was rated high and the other was rated low in frequency. The mean rating of the high-frequency group was 4.23 and that of the low-frequency group was 1.69. Although in the voweled form these consonant strings were unequivocally specified, since their consonantal structures were shared by different words, we labeled the critical pairs as ambiguous and differentiated between voweled and unvoweled ambiguous words.

The unambiguous words were 32 consonant strings, each of which represents in print only one word. Sixteen of the 32 unambiguous words were high-frequency and 16 were low-frequency, according to the same criteria as above. Rating was performed by a different group of $\mathbf{5 0}$ judges in a similar manner (Frost, Katz, \& Bentin, in press). The mean rating was 4.18 for the high-frequency unambiguous group and 1.71 for the low-frequency unambiguous group.

The nonwords in the optional condition were 32 pronounceable but meaningless permutations of consonants arbitrarily assigned with vowel marks. We shall label these stimuli regular nonwords. Note that regular nonwords could not be read as words even if presented unvoweled. In contrast, in the obligatory condition, the nonwords were in fact words when unvoweled or when voweled correctly; the consonantal patterns corresponded to words, but in this study they were inappropriately voweled. These nonwords were labeled special nonwords. The special nonwords were generated from words of average frequency.

All stimuli were generated by a PDP-11/34 computer and displayed at the center of a Tektronix CRT. The size of each letter was $1.2 \times 1.2 \mathrm{~cm}$, and the length of the whole word was between 4.5 and $6.5 \mathrm{~cm}$ (three to five letters), subtending a visual angle of approximately $4.5^{\circ}$.

Design. Six test lists were assembled. In Lists A to D, all stimuli were presented with the vowel marks. In Lists $E$ and F, the stimuli were presented in the regular unvoweled manner. Lists A and B were presented in the optional condition. List A comprised the 16 high-frequency alternatives of the ambiguous pairs, the $16 \mathrm{low}-$ frequency unambiguous words, and the 32 regular nonwords. List B comprised the 16 low-frequency alternatives of the ambiguous pairs, the 16 high-frequency unambiguous words, and the same 32 nonwords used in List $A$. Lists $C$ and D were presented in the obligatory condition. List $\mathbf{C}$ comprised the same words as List $\mathrm{A}$, but the set of 32 special nonwords was employed. List D comprised the same words as List B and the special nonwords. Lists E and F were similar to Lists A and B, respectively, but words were presented without the vowel marks. Therefore, Lists $E$ and $F$ included identical ambiguous words (because the different alternatives of the ambiguous words were indistinguishable without the vowel marks) but different unambiguous words. Different groups of 16 subjects each were randomly assigned to each of these lists.

Procedure. The experiment took place in a semidarkened soundtreated room. Subjects sat approximately $70 \mathrm{~cm}$ from the screen. They were instructed to press one of two alternative microswitch buttons, according to whether the stimulus on the screen was or was not an actual Hebrew word. The dominant hand was always used for the "yes" (i.e., word) responses and the other hand for the "no" (i.e., nonword) responses. Subjects presented with Lists C and $\mathrm{D}$ were warned about the special nature of the nonwords.

Following the instructions, 32 practice trials (16 words and 16 nonwords) were presented. Words/nonwords on the practice trials

\begin{tabular}{|c|c|c|c|}
\hline & $\begin{array}{c}\text { High Frequency } \\
\text { Alternotive }\end{array}$ & $\begin{array}{c}\text { Low Frequency } \\
\text { Alternative }\end{array}$ & $\begin{array}{c}\text { Unvoweled } \\
\text { Print }\end{array}$ \\
\hline $\begin{array}{l}\text { Graphemic } \\
\text { representation }\end{array}$ & דָנָי & דִבְּוּ & רבו \\
\hline $\begin{array}{l}\text { Phonetic } \\
\text { transcription }\end{array}$ & davar & dever & $?$ \\
\hline $\begin{array}{l}\text { English } \\
\text { translation }\end{array}$ & thing & pest & $?$ \\
\hline
\end{tabular}

Figure 1. Example of ambiguity in Hebrew print. Note that the two words represented by the same consonant string are not homophones. 
were prepared in congruence with those on the test list that followed. The 64 test trials were presented next at a rate of one stimulus every $2.5 \mathrm{sec}$. The subject's response terminated the stimulus exposure. If no response was given within $2 \mathrm{sec}$, the stimulus was removed and an error was marked. The subject started the test trials by pressing a "ready" button. RTs were measured in milliseconds, and errors were marked.

\section{Results}

The RTs for correct responses were averaged for each word over the 16 subjects who were exposed to it, and for each subject over the 16 words in each frequency group. RTs that were above or below two standard deviations from a subject's or a word's mean were excluded, and the mean was recalculated. Less than $1.5 \%$ of the RTs were outliers.

We will describe first the comparison between the ambiguous consonant strings in the unvoweled and the two voweled presentations. The RTs to the high-frequency words in List A, to the low-frequency words in List B, and to the unvoweled ambiguous words in List $\mathrm{E}^{2}$ were compared by a one-way ANOVA ${ }^{3}$ and the Tukey-A post hoc procedure. RTs to unvoweled consonant strings were faster than to any of the two voweled alternatives, and RTs to the high-frequency alternatives were faster than to the low-frequency alternatives (Figure 2). The ANOVAs revealed that all differences were significant [for the stimulus analysis, $F(2,30)=22.09, M S e=4,521$, $p<.0001$; for the subject analysis, $F(2,45)=5.15, M S \mathrm{e}$ $=14,700, p<.01$; and $\left.\min F^{\prime}(2,63)=4.18, p<.05\right]$.

\section{AMBIGUOUS WORDS}
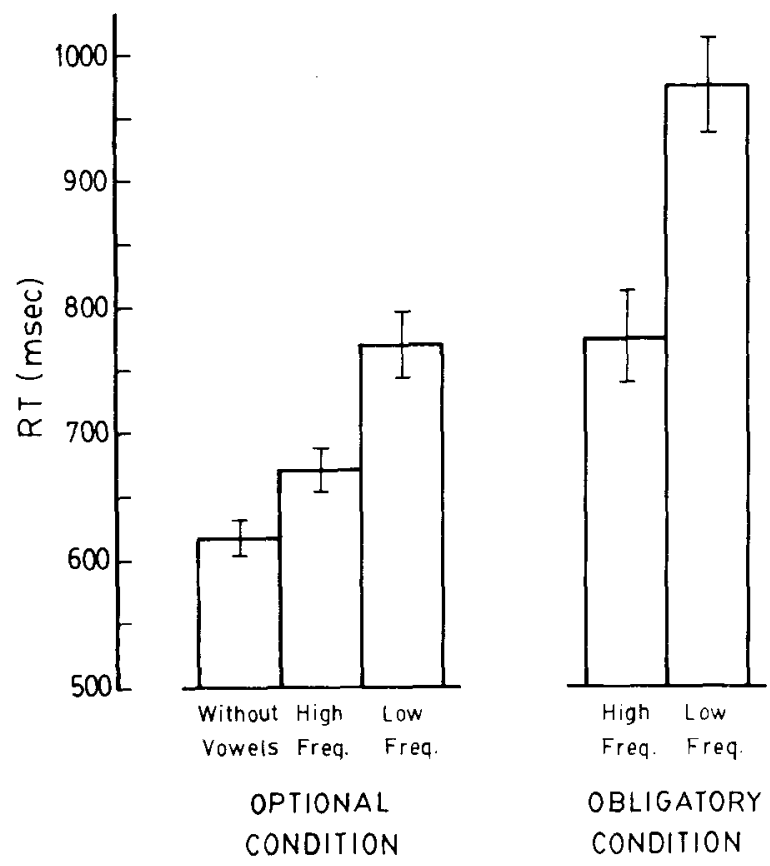

Figure 2. Mean reaction times and standard errors to ambiguous words in the lexical decision task. In the obligatory condition, nonwords would become real words if presented without the vowel marks.

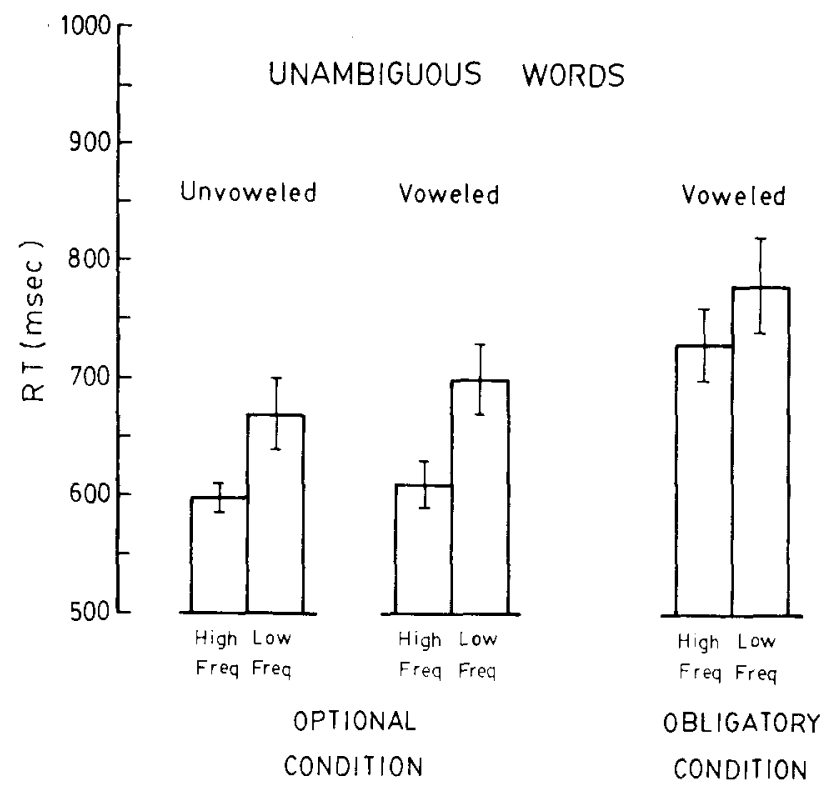

Figure 3. Mean reaction times and standard errors to unambiguous words in the lexical decision task. In the obligatory condition, nonwords would become real words if presented without the vowel marks.

The effect of the special nonwords on lexical decisions for high- and low-frequency voweled ambiguous words were assessed by comparing the RTs to ambiguous words in the optional condition (List A and List B) and in the obligatory condition (List $\mathrm{C}$ and List $\mathrm{D}$ ) in a two-way (condition $\times$ frequency) ANOVA. RTs to ambiguous words were slower in the obligatory condition than in the optional condition $\left[\min F^{\prime}(1,24)=10.96, p<.01\right]$. Across conditions, RTs to high-frequency words were faster than to low-frequency words $\left[\min F^{\prime}(1,49)=9.97\right.$, $p<.01]$. However, the most important result of this comparison was that the frequency effect was twice as large in the obligatory condition as in the optional condition (Figure 2). This interaction was significant both for the stimulus analysis $[F(1,15)=11.91, M S e=3,346$, $p<.004]$ and for the subject analysis $[F(1,60)=4.25$, $M S \mathrm{e}=92,036, p<.05]\left[\min F^{\prime}(1,73)=3.13\right.$, $p<.06$.

The effect of the vowel marks on high- and lowfrequency unambiguous words was assessed by comparing the voweled high-frequency unambiguous words in List $B$ and the voweled low-frequency unambiguous words in List $\mathbf{A}$, with their unvoweled presentations in Lists $E$ and $F$, respectively (Figure 3). A two-way (vowel condition $\times$ frequency) ANOVA revealed a significant frequency effect $[F(1,60)=7.44, M S e=12,334$, $p<.01]$, but no effect of the vowel marks and no interaction.

The effect of the special nonwords on lexical decisions for voweled unambiguous words was assessed by comparing the RTs to voweled high- and low-frequency unambiguous words in the optional condition (List B and List $\mathrm{A}$ ), and in the obligatory condition (List D and 
List C). A condition $x$ frequency two-way ANOVA revealed that lexical decisions were slower in the obligatory than in the optional condition $[F(1,60)=9.48, M S e$ $=18,471, p<.005]$ and slower for low-frequency than for high-frequency words $[F(1,60)=3.84, M S e=$ $18,471, p<.05]$, but in contrast to ambiguous words, there was no interaction between the two factors $[F(1,60)$ $=0.31]$. The different effect of nonword condition on ambiguous and unambiguous words was verified by an ambiguity (ambiguous, unambiguous) $\times$ condition (optional, obligatory) $\times$ frequency (high, low) ANOVA, revealing a marginally significant three-way interaction $[F(1,120)=3.57, M S e=20,067, p<.06]$.

The nonwords in Lists A and B were identical, as were the nonwords in Lists $C$ and $D$ and those in Lists $E$ and $F$. Therefore, the RTs to nonwords in the optional condition (Lists A and B) were compared with the RTs to nonwords in the obligatory condition (Lists $\mathrm{C}$ and $\mathrm{D}$ ) and with RTs in unvoweled nonwords (Lists $\mathrm{E}$ and $\mathrm{F}$ ) by a oneway ANOVA and Tukey-A post hoc procedure. ${ }^{4}$ RTs to voweled nonwords in the optional condition and to unvoweled nonwords were not significantly different ( $764 \mathrm{msec}$ and $739 \mathrm{msec}$, respectively), and both were faster than the RTs to nonwords in the obligatory condition $(1,055 \mathrm{msec})[F(2,93)=36.16, M \mathrm{Se}=27,255$, $p<.0001$.

The distribution of errors between the different stimulus groups and conditions is presented in Table 1. Although the uneven distribution of errors within cells precluded a significant statistical analysis, the overall pattern does not suggest a speed-accuracy trade-off between conditions.

\section{Discussion}

The comparison of responses to voweled and unvoweled ambiguous words revealed that explicit presentation of the vowel marks that disambiguated the consonant strings did not facilitate lexical decisions relative to decisions about the ambiguous unvoweled strings. In fact, an opposite effect was found. Apparently, this result simply replicates previous findings that suggested that lexical decisions for homographs are faster than those for nonhomographs.
However, we have obtained this result with consonant strings that represented in print not only different meanings, but also different words (i.e., different phonological representations). Therefore, our results are in contrast to results obtained in similar studies about English (Kroll \& Schweickert, 1978).

There are several ways to explain our data. The first explanation is based on the hypothesis that a lexical decision for an ambiguous unvoweled consonant string is based on accessing at least one of the words it represents. The existence of several possible entries increases the probability that one of them will be accessed. This explanation is unlikely for two reasons: (1) in English this effect was found only for homographs that represented different meanings but only one pronunciation; (2) the probabilistic explanation is based on the assumption that the distribution of the RTs to the different meanings represented by the homograph partially overlap. Although this is probably true in our study as well, we tried to diminish this overlap by selecting only homographs that represented both very high-frequency and very lowfrequency words.

The second explanation of our data is based on the assumption that Hebrew readers are more familiar with unvoweled than with voweled words. This explanation, however, is contradicted by the absence of an effect of vowel marks on lexical decisions for unambiguous words and for nonwords.

The third explanation is congruent with our hypothesis. If lexical decisions for ambiguous consonant strings do not require phonological disambiguation, addition of vowel marks is unnecessary. Moreover, vowel marks add information that probably cannot be totally ignored and thus increases the word processing time. Therefore, we suggest that these data support the hypothesis that lexical decisions for unvoweled Hebrew homographs may be based on information that is common to all lexical alternatives that are represented by one consonant string. Decisions based on the common information should be at least as fast as those based on accessing specific entries, for the additional reason that the frequency of the common consonant string is higher than the frequency of each

Table 1

Mean Percent of Errors and Standard Error of the Mean (SEM) in Lexical Decision for Voweled and Unvoweled, Ambiguous and Unambiguous Words and Nonwords in the Optional and Obligatory Conditions

\begin{tabular}{|c|c|c|c|c|c|c|c|c|c|}
\hline & \multicolumn{3}{|c|}{ Ambiguous Words } & \multicolumn{4}{|c|}{ Unambiguous Words } & & \\
\hline & \multicolumn{2}{|c|}{ Voweled } & \multirow[b]{2}{*}{ Unvoweled } & \multicolumn{2}{|c|}{ Voweled } & \multicolumn{2}{|c|}{ Unvoweled } & \multicolumn{2}{|c|}{ Nonwords } \\
\hline & Hi Freq & Lo Freq & & Hi Freq & Lo Freq & Hi Freq & Lo Freq & Voweled & Unvoweled \\
\hline \multicolumn{10}{|c|}{ Optional Condition } \\
\hline Percent Errors & 3.19 & 13.67 & 1.17 & 2.73 & 6.25 & 3.13 & 11.33 & 3.12 & 6.25 \\
\hline SEM & 1.22 & 1.76 & 0.61 & 1.10 & 1.56 & 1.10 & 1.85 & 1.26 & 1.42 \\
\hline \multicolumn{10}{|c|}{ Obligatory Condition } \\
\hline Percent Errors & 9.66 & 14.50 & & 2.34 & 8.20 & & & 14.35 & \\
\hline SEM & 1.84 & 2.90 & & 0.87 & 2.39 & & & 2.05 & \\
\hline
\end{tabular}


of its individual lexical realizations. This suggestion is supported by previous results, which revealed that lexical decisions for low-frequency ambiguous consonant strings are faster than for low-frequency unambiguous consonant strings (Bentin et al., 1984).

Word frequency affected lexical decision performance in both the optional and the obligatory conditions, suggesting that subjects did not ignore the differences between the words even if such a detailed analysis was not absolutely necessary for lexical access. Apparently, this result suggests that when the element of ambiguity is eliminated (even by adding unfamiliar vowels), lexical decisions are based on a full analysis of the graphemic and the phonemic codes. However, the enhanced effect of word frequency in the obligatory condition suggests that such a simple conclusion might be premature. Recall that in the optional condition discrimination between words and nonwords could have been accomplished even if the vowels were ignored. Therefore, a relatively reduced effect of frequency may have resulted if some of the decisions were based only on the consonant strings (which were identical in the high-and low-frequency groups), whereas other decisions involved processing of the full phonological code. However, an alternative explanation is possible. The overall slower RTs in the obligatory than in the optional condition suggest that the word/nonword discrimination was more difficult in the former than in the latter condition. Obviously, the words and the nonwords in the obligatory condition were more alike, reducing the certainty level of the subjects. The uncertainty could have been greater for the low- than for the high-frequency words since the former were subjectively more similar to nonwords. This interpretation is supported by previous studies that revealed that low-frequency word decisions are facilitated more than high-frequency word decisions by the essence of unpronounceable nonwords (Duchek \& Neely, 1984, cited by Balota \& Chumbley, 1984; James, 1975). Thus, the interaction between the effect of frequency and the nature of the nonwords may be explained as a postaccess decision factor rather than a different manner of lexical access.

An insight into the origin of the interaction between the nonword type and the word frequency effect can be achieved by comparing the condition effect on the ambiguous words with the effect on the unambiguous words. Recall that, for unambiguous words, the word frequency effect did not interact with the effect of the nonword type. If the difference in the magnitude of the frequency effect in the obligatory and optional conditions was not related to word ambiguity, it should have emerged with unambiguous words as well. Therefore, we conclude that the interaction between the nonword type and the frequency effect was related to the ambiguity factor. Although other explanations are possible, we propose to consider this interaction as corroborative evidence that lexical decisions for ambiguous Hebrew words do not require phonological disambiguation.

\section{EXPERIMENT 2}

In contrast to making lexical decision, naming a phonemically ambiguous string of consonants necessarily requires the selection of only one of the alternative phonological representations. The main purpose of Experiment 2 was to investigate the nature of this selection in an attempt to enhance our understanding of the process of disambiguation of Hebrew unvoweled consonant strings.

Previous studies in Hebrew employed only unambiguous words, that is, consonant strings that represented only one lexical item and could be pronounced correctly in only one manner. It has been reported that naming voweled words was delayed when the vowel marks were incompatible with the correct sound of the word, even if the subjects were instructed to ignore the vowels, but it was equally fast if unvoweled words were compared with correctly voweled words (Navon \& Shimron, 1981). In a more recent study, however, vowels were found to speed up naming, although they had no effect on lexical decisions (Koriat, 1984).

Recent data from our laboratory revealed that in contrast to more shallow orthographies (such as SerboCroatian and English), in Hebrew, making lexical decisions for unvoweled unambiguous consonant strings was faster than reading the same words aloud (Frost et al., in press). Furthermore, significant semantic priming was found for naming in Hebrew, but not in Serbo-Croatian. These results suggest that although naming voweled Hebrew words can, in principle, be based on phonetic cues generated via a process of grapheme-to-phoneme transformation, naming unvoweled words is always mediated by the lexicon. Therefore, lexical ambiguity would influence naming because it probably requires a choice among several lexical representations. In the present experiment, we compared the naming of ambiguous and unambiguous voweled and unvoweled stimuli. This comparison, we hoped, would shed additional light on naming words in a deep orthography in general, and on the rules of disambiguation of Hebrew homographs in particular.

An additional aspect of word processing that might be influenced by lexical ambiguity is the word frequency effect on naming. Several studies suggested that frequency effects are smaller in naming than in lexical decision tasks (Andrews, 1982; Balota \& Chumbley, 1984; Frederiksen \& Kroll, 1976). The same relationship was recently found in Hebrew (Frost et al., in press). In that study, however, only unambiguous words were employed. Naming ambiguous words, on the other hand, might be affected by frequency both during lexical access and at postlexical processing stages (Balota \& Chumbley, 1985; Forster \& Bednall, 1976; Simpson, 1981). Therefore, it is possible that naming voweled ambiguous words might be affected more strongly by the relative frequency of their alternative phonological representations than naming voweled high- and low-frequency unambiguous words. 
A secondary purpose of Experiment 2 was to test this hypothesis.

\section{Method}

Subjects. The subjects were 64 undergraduates who were paid for their participation. They were all native speakers of Hebrew, with normal or corrected-to-normal vision. None of them had participated in Experiment 1.

Stimuli and Design. The stimuli were identical to those used in Experiment 1 . Only List A (voweled high-frequency ambiguous words, low-frequency unambiguous words, and regular nonwords), List B (voweled low-frequency ambiguous words, high-frequency unambiguous words, and regular nonwords), and Lists $E$ and $F$ (unvoweled replicas of Lists A and B, respectively) were used, each presented to a different group of 16 subjects. The assignment of subjects to lists was random.

Procedure. The conditions of Experiment 1 were repeated in this experiment. The subjects were instructed to read aloud as fast as possible words and nonwords that were presented to them on the CRT screen. In the voweled condition (Lists A and B), subjects were told to read the stimuli as voweled. In the unvoweled condition (Lists $\mathrm{E}$ and $\mathrm{F}$ ), subjects were told to read aloud as fast as possible the words and the nonwords that were presented on the screen. Since, in Hebrew, reading without vowels is the rule rather than the exception, no additional instructions were given (or solicited) for reading the words. Subjects were told, however, that there was no correct or incorrect way to read the nonwords, and that they could pronounce them by arbitrary assignment of vowels to the consonants.

Subjects' responses were recorded by Mura DX-118 microphone, which was connected to a Colbourne Instruments voice-key. RTs were measured in milliseconds from stimulus onset by the computer; responses were recorded on a magnetic tape for off-line analysis.

\section{Results}

As in Experiment 1, the RTs were averaged across subjects for each word and across words for each subject.
All RTs were normalized by excluding responses that were above or below two standard deviations from the subject's or the word's mean. Less than $1.5 \%$ of the RTs were excluded.

The time to initiation of naming ambiguous consonant strings in the high- and low-frequency presentations (Lists $A$ and B, respectively) and in the unvoweled condition (List E) ${ }^{5}$ was analyzed by one-way ANOVAs across stimuli and across subjects (see Note 3 ). In contrast to lexical decision performance, the unvoweled consonant strings were named as fast as the high-frequency voweled alternatives, but both groups were named faster than the low-frequency alternatives (Figure 4A). This pattern of performance was supported by the ANOVAs followed by Tukey-A post hoc comparisons [for the stimulus analysis, $F(2,30)=25.79, M S e=2,268, p<.0001 ;$ for the subject analysis, $F(2,45)=5.40, M S e=12,679, p<.008$; $\left.\min F^{\prime}(2,65)=4.46, p<.025\right]$

The comparison between voweled and unvoweled unambiguous words is presented in Figure 4B. Statistical significance of the differences was assessed by vowels (voweled, unvoweled) $x$ frequency (high, low) ANOVAs across stimuli and across subjects. Unvoweled words were named $30 \mathrm{msec}$ faster than voweled words. This difference was significant for the stimulus analysis $[F(1,30)=$ $16.47, M S e=13,718, p<.001]$, but not for the subject analysis $[F(1,30)<1]$. Consequently, the $\min F^{\prime}$ was not significant. The high-frequency words were named $26 \mathrm{msec}$ faster than the low-frequency words. This difference was not significant either for the subject or for the stimulus analysis. The interaction between frequency and vowel conditions was not significant.

None of the unvoweled words was erroneously read as

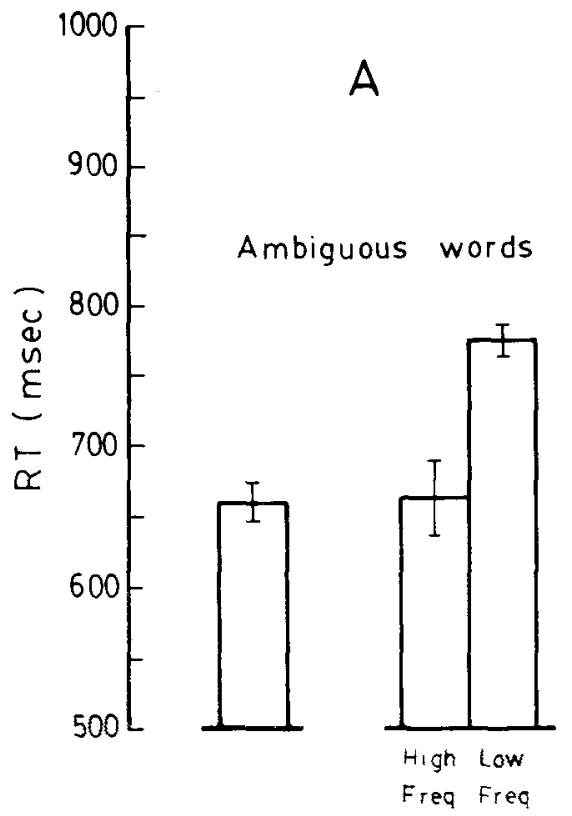

Unvoweled

Voweled

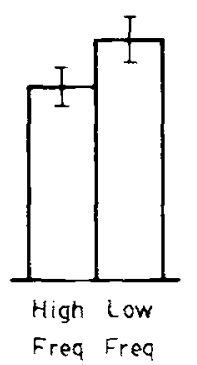

Unvoweled

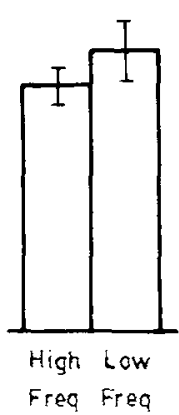

Voweled

Figure 4. Mean reaction times and standard errors to voweled and unvoweled words in the naming task. $A=$ ambiguous words. $B=$ unambiguous words. 
a nonword, and except four occasional errors all voweled words were read correctly. All four errors were on lowfrequency alternatives. For technical reasons, errors made while reading voweled nonwords could not be recovered from the raw data.

The analysis of the words that were actually pronounced by each subject, when unvoweled ambiguous words were presented, revealed that, for 4 of 16 consonant strings the high-frequency alternative was unanimously chosen by 32 subjects, and for 8 strings the high-frequency alternative was pronounced by more than $75 \%$ of the subjects, and in no case was the high-frequency alternative chosen by less than $45 \%$ of the subjects. In contrast, 7 low-frequency alternatives were not chosen by any subject, and 7 were pronounced by less than $10 \%$ of the subjects, whereas none of the low-frequency alternatives was chosen by more than $40 \%$ of the subjects.

The nonwords in the voweled condition (List A and List B) and in the unvoweled condition (List E and List F) were compared by a $t$ test. In contrast to lexical decisions, naming of unvoweled nonwords was significantly faster than naming of voweled nonwords [757 msec vs. $843 \mathrm{msec} ; t(62)=2.24, p<.03$ ].

\section{Discussion}

The naming-time data revealed that the only significant difference between naming voweled and unvoweled words was the slower naming of the voweled low-frequency alternatives of ambiguous words relative to naming of the unvoweled alternatives. In addition, the frequency effect for naming ambiguous voweled words $(99 \mathrm{msec})$ was as large as the frequency effect in the lexical decision task and considerably larger than that for naming unambiguous voweled words $(16 \mathrm{msec})$. Note that for voweled unambiguous words, the relationship between frequency effects in naming and lexical decision tasks replicates our findings with Hebrew unvoweled unambiguous words, as well as findings from previous studies in English.

These data suggest that vowel marks did not facilitate the naming of printed words. Moreover, vowel marks interfered with pronunciation when they imposed an unexpected interpretation of the grapheme. Therefore, we propose that the subjects might have initially generated a phonological code on the basis of the consonantal information. In the case of ambiguous words, the most frequent alternative was probably activated. A subsequent consideration of the vowel marks had no significant effect on the processing time if they were congruent with the subject's initial response tendency (as was the case with the high-frequency alternatives or with the unambiguous words), but vowel marks required a timeconsuming revision of the output pattern if they were incongruent with the initial response. Supporting this hypothesis, all subjects chose the high-frequency alternative in most trials while naming unvoweled consonant strings.

This interpretation assumes that the enhancement of the frequency effect for naming ambiguous words, relative to that for naming unambiguous words, originates from postaccess processing. In agreement with Forster (1981; see also Kinoshita, 1985), we suggest that the delay in naming voweled low-frequency ambiguous words reflects the time spent in evaluating the initially generated highfrequency phonology vis-à-vis the presented vowels and rejecting this alternative in favor of the low-frequency phonology.

\section{GENERAL DISCUSSION}

In this study, we investigated the process of disambiguation of phonemically and semantically ambiguous Hebrew printed words and the effects of this deep orthography on lexical decision and naming performance. The results suggest that when unvoweled consonant strings are presented, lexical decisions are based primarily on the ambiguous grapheme; lexical disambiguation is achieved in parallel but has little influence on the decision processes per se. In contrast, phonemic (and therefore semantic) disambiguation must precede naming of unvoweled consonant strings, and we suggest that the process of disambiguation is based on a postaccess race of the different phonemic/semantic lexical representations to which the specific consonant string is related. The result of this race (i.e., the word that is pronounced) is determined to a great extent by the relative frequency of the alternative lexical representations. These conclusions are based on the following pattern of observations.

\section{Stimulus Ambiguity and Lexical Decision}

Presentation of the vowel marks in conjunction with the consonant letters disambiguates the printed Hebrew word. Even though word perception should have been facilitated by exclusion of the ambiguity factor, our data revealed that addition of vowel marks significantly delayed lexical decisions for ambiguous words. The direct implication of this result is that, in Hebrew, the information provided by the vowels is not absolutely necessary for lexical decisions. A possible explanation is that the lexical decision for unvoweled homographs is normally based on the ambiguous string of letters that may be recognized as a word (or rejected) without access to any of the alternative meanings; phonological disambiguation is, according to this hypothesis, a postaccess process. This hypothesis implies that lexical decisions for unvoweled ambiguous Hebrew words are made without reference to their meaning and, therefore, apparently contradicts the wellestablished effects of word meaning on lexical decisions (e.g., the semantic priming effect). We can, however, account for this apparent contradiction by assuming, along with Chumbley and Balota (1984), that the effect of word meaning in lexical decision is attributable to a decision stage following lexical access. We will elaborate our view within the framework of a slightly modified version of the two-stage model of lexical decision performance proposed by Balota and Chumbley (1984). Briefly, this model assumes that letter strings (words and nonwords) differ on a familiarity/meaningfulness (FM) dimension. The 
value of a particular letter string on the FM dimension is determined by its orthographic and phonological similarity to real words. Strings with very high or very low FM values are classified as words and as nonwords, respectively, during a first stage of the decision process. If the computed FM value is not extreme, a second stage, in which a more detailed analysis of the stimulus is accomplished, determines the decision. Obviously, the distribution of the FM values for words and nonwords overlaps. The amount of overlap is related to the discriminability between words and nonwords in the particular stimulus list.

The relative contributions of the phonological/semantic meaningfulness and orthographic familiarity to the computation of the FM value was not specified by the twostage model. In agreement with Balota and Chumbley (1984), we suggest that the analyses of the orthographic familiarity and of the meaningfulness of the stimulus overlap in time to a great extent. However, the determination of an FM value need not wait until both analyses are exhausted; whenever enough information accumulates, regardless of its source (orthographic or phonologic/semantic), a value is set. The relative contribution of each type of analysis depends on the familiarity of the orthographic cluster and on the availability of its meaning. The orthographic familiarity of homographs is enhanced because the cluster of consonants is encountered in several semantic contexts; on the other hand, their meaning is ambiguous and, therefore, not immediately available. Consequently, we suggest that the FM value for homographs is based mostly on the orthographic, rather than on the semantic/phonological, familiarity. Therefore, whenever the lexical decision is based on the first-stage computation of the FM value, it is done before the phonology and meaning of the homograph are disambiguated. Furthermore, we suggest that this strategy is employed for most unvoweled words, since even for single-meaning unvoweled words, the phonology is not immediately available in print. Empirical support for this last hypothesis was provided in a previous study (Bentin et al., 1984). ${ }^{6}$

The interference effect of vowel marks on lexical decision suggests that, when vowels are present, subjects cannot ignore them. This hypothesis is strongly supported by the significant frequency effect observed when the voweled high- and low-frequency alternatives of the ambiguous consonant strings were compared. It is possible that the addition of vowels reduced the orthographic familiarity of the stimuli and, at the same time, guided the retrieval of the meaning. Therefore, the computation of FM values was based on both orthographic and phonologic/semantic analysis. Alternatively, the FM value was determined by the orthographic analysis, and the meaning was determined during the second-stage analysis. The second possibility implies that lexical decisions for voweled words require the complete two-stage analysis in many more cases than required for unvoweled words. This hypothesis might also explain the observed difference between voweled and unvoweled words. Note, however, that even if this explanation is true, it does not invalidate our claim that for unvoweled words, and particularly for homographs, the lexical decision is based mostly on the first-stage analysis, which does not include phonological disambiguation.

The only difference between the optional and the obligatory conditions was that the orthographic similarity between words and nonwords was significantly larger in the obligatory condition. If, indeed, the FM value is determined primarily by orthographic familiarity, this manipulation should have shifted the nonword distribution along the FM value to the right, increasing its overlap with the distribution of words. Following the logic underlying Balota and Chumbley's (1984) model, this manipulation should have forced the subjects to increase the upper criterion above which a word is accepted without further analysis. Since most low-frequency words are located below the original criterion, raising this criterion should have affected the high- more than the lowfrequency words, and, therefore, the net effect should have been an attenuation rather than an amplification of the frequency effect. This trend was indeed observed with unambiguous words; the frequency effects in the obligatory condition $(42 \mathrm{msec})$ were smaller than those in the optional condition (67 msec) (see Table 2). However, the same manipulation with ambiguous words yielded opposite results: the frequency effect in the obligatory condition $(201 \mathrm{msec})$ was twice as large as that in the optional condition (102 msec) (Table 2). One possible explanation of this result is that vowel marks have a larger effect when they indicate a low-frequency rather than a high-frequency

Table 2

Summary of Reaction Times (in Milliseconds) in the Lexical Decision and Naming Experiments

\begin{tabular}{|c|c|c|c|c|c|c|c|c|}
\hline \multicolumn{3}{|c|}{ Ambiguous Words } & \multicolumn{4}{|c|}{ Unambiguous Words } & & \\
\hline \multicolumn{2}{|c|}{ Voweled } & \multirow[b]{2}{*}{ Unvoweled } & \multicolumn{2}{|c|}{ Voweled } & \multicolumn{2}{|c|}{ Unvoweled } & \multicolumn{2}{|c|}{ Nonwords } \\
\hline Hi Freq & Lo Freq & & Hi Freq & Lo Freq & Hi Freq & Lo Freq & Voweled & Unvoweled \\
\hline \multicolumn{9}{|c|}{ Lexical Decision: Optional Condition } \\
\hline 670 & 772 & 616 & 635 & 702 & 610 & 678 & 764 & 739 \\
\hline \multicolumn{9}{|c|}{ Lexical Decision: Obligatory Condition } \\
\hline 775 & 976 & & 748 & 790 & & & 1,055 & \\
\hline \multicolumn{9}{|c|}{ Naming } \\
\hline 669 & 768 & 653 & 674 & 690 & 634 & 671 & 843 & 757 \\
\hline
\end{tabular}


alternative of an ambiguous consonant string. Thus, a new aspect of the disambiguation process is disclosed: while processing a letter string, subjects might automatically generate possible lexical representations of the grapheme. When the letter string is voweled, generation of phonology might be initially based only on consonants, independently of the specific vowels employed, because the subjects have little experience with reading vowels, and because the consonants are visually more salient. At some stage, however, the top-down-generated lexical candidate (which provides unequivocal meaning and phonology) is confronted with the bottom-up analysis of the vowels. We suggest that, at this stage, vowels that indicate a highfrequency lexical alternative have a different effect than those that indicate a low-frequency word. We assume that top-down generation of meanings is influenced by frequency. High-frequency words are more readily available and, therefore, are generated first in a sequential or cascade-type process. Therefore, the lexical candidate that is first confronted with the bottom-up vowel information is a high-frequency alternative. If the vowels indicate a different word, the subject must reject his or her first hypothesis, and generate (or at least consider) another one. This hypothesis, which is not basically different from the postaccess inhibition model suggested for naming by Forster (1981), is also supported by the naming performance in Experiment 2.

\section{Stimulus Ambiguity and Naming}

In the naming task, we were able to know which alternative was chosen when an unvoweled ambiguous consonant string was presented. The data revealed that the high-frequency alternatives were indeed chosen by the great majority of subjects. We have no immediate explanation for those few cases in which low-frequency alternatives were selected, but we tend to believe that these selections were caused by coincidental circumstances, such as unusual individual preferences or phonetic priming by the previous random stimulus. At any rate, we consider the analysis of the overt responses as supporting the word-frequency-guided order of meaning generation for ambiguous letter strings.

The effect of vowel marks on naming was very different from their effect on lexical decision. Unvoweled ambiguous words were named significantly faster than the low-frequency alternatives but were named only $16 \mathrm{msec}$ faster than were the voweled high-frequency alternatives. Unambiguous words were named $30 \mathrm{msec}$ faster if they were presented without the vowel marks than if they were voweled. This difference was small relative to the intersubject variability and, therefore, was not significant. However, the direction of the difference conflicts with the results of Koriat (1984). One difference between the two studies is that Koriat employed only unambiguous words. We do not have a simple model to explain how this difference might have affected naming performance, but it seems to us that the reason for the discrepant results should be related to this difference between the stimulus lists.
The main difference between lexical decision and naming unvoweled strings is that naming cannot be performed unless the stimulus is phonologically disambiguated. Because the print does not provide enough phonemic cues, naming requires postaccess processes of disambiguation. Therefore, in contrast to other languages, in Hebrew, whenever lexical decision requires second-stage processes (as, for example, when vowel marks are presented), semantic ambiguity should affect both tasks in a similar way. This assumption is supported by the remarkable similarity of the frequency effect and on absolute naming time and lexical decision time in the optional condition. On the other hand, when lexical decisions can be based on an orthographically generated FM value, naming is relatively delayed.

In conclusion, we suggest that the results of this study revealed that phonological disambiguation of Hebrew unvoweled words does not occur prelexically. Furthermore, at least for Hebrew consonant strings, it appears that lexical decisions are based on the ambiguous orthographic information without reference to meaning or phonological structure. We propose that multiple meanings facilitate lexical decisions by increasing orthographical familiarity and that the decisions are therefore based on this factor alone. These processes are best explained in the context of a multistage model of visual word recognition, such as the two-stage model proposed by Balota and Chumbley (1984), with only slight modifications and additions.

It is difficult to comment on the generality of these hypotheses for languages other than Hebrew. Recall, however, that Jastrzembski (1981) reported that among words with an equal number of derivations and an equal number of meanings, those whose meanings tend to be associated with only one derivation were responded to faster. Furthermore, Chumbley and Balota (1984) revealed that lexical decision RTs and RTs in semantic tasks are closely related, independent of other factors. If indeed RTs in semantic association tasks and clustering of meanings around only one etymological derivation are measures of meaning availability, these results suggest that our findings in the deep Hebrew orthography are a rather extreme example of processing ambiguity in printed words.

\section{REFERENCES}

ANDREws, S. (1982). Phonological recording: Is the regularity effect consistent? Memory \& Cognition, 10, 565-575

Balota, D. A., \& Chumbley, J. I. (1984). Are lexical decisions a good measure of lexical access? The role of word frequency in the neglected decision stage. Joumal of Experimental Psychology: Human Perception \& Performance, 10, 340-357.

Balota, D. A., \& Chumbuey, J. I. (1985). The locus of word-frequency effects in the pronunciation task: Lexical access and/or production? Joumal of Memory \& Language, 24, 89-106.

Bentin, S., BArgal, N., \& Katz, L. (1984). Orthographic and phonemic coding for lexical access: Evidence from Hebrew. Joumal of Experimental Psychology: Leaming, Memory, \& Cognition, 10, 353-368. 
Bentin, S., \& Katz, L. (1984). Semantic awareness in a nonlexical task. Bulletin of the Psychonomic Society, 22, 134-139.

Carpenter, P. A., Daneman, M. (1981). Lexical retrieval and error recovery in reading: A model based on eye fixation. Journal of Verbal Learning \& Verbal Behavior, 20, 137-161.

Chumbley, J. I., \& Balota, D. A. (1984). A word's meaning affects the decision in lexical decision. Memory \& Cognition, 12, 590-606.

FORSTER, K. I. (1981). Priming and the effects of sentence and lexical contexts on naming time: Evidence for autonomous lexical processing. Quarterly Journal of Experimental Psychology, 33A, 465-496.

Forster, K. I., BEDNALL, E. S. (1976). Terminating and exhaustive search in lexical access. Memory \& Cognition, 4, 53-61.

Frederucsen, J. R., Kroll, J. F. (1976). Spelling and sound: Approaches to the internal lexicon. Joumal of Experimental Psychology: Human Perception \& Performance, 2, 361-379.

Frost, R., KATZ, L., BENTIN, S. (in press). Strategies for visual word recognition and orthographical depth: A multilingual comparison. Journal of Experimental Psychology: Human Perception \& Performance.

JaMES, C. T. (1975). The role of semantic information in lexical decisions. Joumal of Experimental Psychology: Human Perception \& Performance, 1, 130-136.

JASTRZEMBSK, J. E. (1981). Multiple meanings, number of related meanings, frequency of occurrence, and the lexicon. Cognitive Psychology, 13, 278-305.

KinOsHiTA, S. (1985). Sentence context effects on lexically ambiguous words: Evidence for postaccess inhibition process. Memory \& Cognition, 13, 579-595.

KorIAT, A. (1984). Reading without vowels: Lexical access in Hebrew. In H. Bouma \& D. G. Bouwhuis (Eds.), Attention and performance $X$ : Control of language processes. Hillsdale, NJ: Erlbaum.

Krol., J. F., \& SCHWEICKERT, J. M. (1978, November). Syntactic disambiguation of homographs. Paper presented at the Nineteenth Annual Meeting of the Psychonomic Society, San Antonio, Texas.

Morton, J. (1970). A functional model for memory. In D. A. Norman (Ed.), Models of human memory. New York: Academic Press.

Morton, J. (1979). Word recognition. In J. Morton \& J. C. Marshall (Eds.), Psycholinguistics 2: Structure and processes. Cambridge, MA: MIT Press.

Navon, D., \& Shimron, J. (1981). Does word naming involve grapheme-to-phoneme translation? Evidence from Hebrew. Journal of Verbal Leaming \& Verbal Behavior, 20, 97-109.

NAYon, D., \& SHimron, J. (1984). Reading Hebrew: How necessary is graphemic representation of vowels? In L. Henderson (Ed.), Orthographies and reading. Hillsdale, NJ: Erlbaum.

Rubenstein, H., Garfield, L., \& Millikan, J. A. (1970). Homographic entries in the internal lexicon. Journal of Verbal Learning \& Verbal Behavior, 9, 487-494.

Rubenstein, H., LewIS, S. S., \& Rubenstein, M. A. (1971). Homo- graphic entries in the internal lexicon: Effects of systematicity and relative frequencies of meanings. Journal of Verbal Learning \& Verbal Behavior, 10, 57-62.

Simpson, G. B. (1981). Meaning dominance and semantic context in the processing of lexical ambiguity. Journal of Verbal Learning \& Verbal Behavior, 20, 120-136.

Simpson, G. B. (1984). Lexical ambiguity and its role in models of word recognition. Psychological Bulletin, 96, 316-340.

\section{NOTES}

1. If the vowel marks are presented in conjunction with the consonants (usually placed below the letters), they disambiguate the phonology and, in most cases, unequivocally determine one word. Thus, in a fully vowelized system, the reader may use the phonemic cues provided by the vowel marks to aid lexical access.

2. Note that the ambiguous consonantal strings in Lists $E$ and $F$ were identical. Therefore, there were twice as many subjects who responded to unvoweled ambiguous words than to each of the voweled alternatives. The RTs to unvoweled consonantal strings in Lists $E$ and $F$ were not significantly different (616 $\mathrm{msec}$ and $621 \mathrm{msec}$, respectively). Therefore, in order to obtain an equal number of observations in each cell, we used only the RTs of the subjects presented with List E.

3. Whenever appropriate, both stimulus and subject analyses were performed, and $\min F^{\prime}$ was calculated. The stimulus analysis used a within-stimulus design, and the subject analysis used a between-subjects design.

4. Initial analysis revealed that, indeed, the RTs to nonwords in List A were similar to those in List B, the RTs to nonwords in List $C$ were similar to those in List D, and the RTs in List E were similar to those in List $\mathrm{F}$.

5. The RTs to ambiguous words in List $E$ and List F were not significantly different ( $653 \mathrm{msec}$ and $665 \mathrm{msec}$, respectively). For technical reasons, the RTs to unvoweled strings were collapsed across the different phonological realizations that were indeed produced. However, since the great majority of responses were high-frequency words, we assume that the average results are not biased significantly.

6. Note, however, that according to our model, stimulus analysis is not terminated by the lexical decision. As we suggested elsewhere (Bentin \& Katz, 1984), words are exhaustively analyzed to the "deepest" lexical level, provided that the task does not interfere with this analysis. Thus, even though the lexical decision was made, the phonological disambiguation continues until at least one (and possibly all) meanings are accessed.

(Manuscript received December 27, 1985; revision accepted for publication July 21,1986 .) 